\title{
Bariatric Surgery Impacts Levels of Serum Lipids during Pregnancy
}

\author{
Latife Bozkurt $^{a} \quad$ Christian S. Göbl ${ }^{b}$ Michael Leutner ${ }^{a} \quad$ Wolfgang Eppel ${ }^{b}$ \\ Alexandra Kautzky-Willer ${ }^{\text {a }}$ \\ ${ }^{a}$ Gender Medicine Unit, Division of Endocrinology and Metabolism, Department of Internal \\ Medicine III, Medical University of Vienna, Vienna, Austria; ${ }^{b}$ Division of Obstetrics and \\ Fetomaternal Medicine, Department of Obstetrics and Gynecology, Medical University of \\ Vienna, Vienna, Austria
}

\author{
Keywords \\ Gastric bypass · Hyperlipidemia · Triglycerides · Pregnancy
}

\begin{abstract}
Introduction: Bariatric surgery confers a high risk for nutritional deficiencies that could affect physiologic adaptation of lipids during pregnancy. We aimed to evaluate differences in serum lipids in pregnant women after bariatric surgery compared to obese and lean mothers. Methods: 25 women with a history of Roux-en-Y gastric bypass (RYGB), 19 obese and 19 normalweight controls were included at the 24th-28th gestational week for determination of fasting lipids with follow-up in a subgroup after delivery. Data on neonatal biometry were additionally assessed. Results: Women after RYGB showed lower total-cholesterol (TC), low-density lipoprotein $C$ (LDL-C), non-high-density lipoprotein $C$ (non-HDL-C) and triglycerides (TG) compared to obese mothers. Despite their higher BMI, women after RYGB showed lower TC, LDL-C and non-HDL-C than normal-weight mothers. Ultrasensitive C-reactive protein was lower in RYGB mothers than in obese ones, reaching values of lean controls. Differences remained unchanged in BMI-matched comparison. Birth weight percentiles of RYGB offspring were associated with maternal TC $(r=0.59, p=0.021)$, LDL-C $(r=0.71, p=0.003)$, non-HDL $(r=0.59, p=0.021)$ but not HDL-C or TG. After delivery, lipids decreased in all women; however, TC and LDL-C showed more attenuated decline in mothers after RYGB than control women. Conclusion: Pregnancies after RYGB show alterations of physiologic patterns in lipid profile. Further studies are required to evaluate whether imbalances in maternal lipids constitute a risk for abnormal fetal growth in this special cohort.


Bozkurt et al.: Lipids in Pregnancy after RYGB

\section{Introduction}

The growing popularity of bariatric surgery in the western world confronts clinicians with various consequences, and these become particularly challenging if women at a childbearing age are affected. Weight loss improves fertility which explains the upcoming trend of increasing numbers of pregnancies after bariatric procedures [1]. Actually, recommendations endorse contraception only during the first 12-18 months after surgery, when weight loss and metabolic changes occur most extensively [1]. Although optimizing prepregnancy weight reduces the risk of obesity-related morbidities (i.e., macrosomia, gestational diabetes, preeclampsia), maternal history of bariatric surgery seems to be associated with occurrence of small-for-gestational-age neonates as well as a tendency for a higher risk of stillbirth or neonatal mortality $[1,2]$. In routine practice metabolic management is focused on glycemic control but in pregnancies after bariatric surgery this strategy is restricted due to physiologic changes that are associated rather with a higher degree of glucose variability and risk for hypoglycemia - altogether factors that challenge the circumstances for diagnosis and therapy options [3]. Thus, the question arises whether it would be favorable for the outcome of pregnancies after bariatric surgery to address additional aspects of maternal metabolism that are affected by the changes in intestinal nutrient absorption. In this regard alterations in maternal lipids might be of relevance as differences in triglycerides (TG) and cholesterol during pregnancy have been associated with maternal obesity and other complications [4].

Since we could previously demonstrate changes in glucometabolic regulation in pregnancies after Roux-en-Y gastric bypass (RYGB) with higher disposition for hypgoglycemia [3, 5], we speculated whether alterations in lipid constellation as another essential substrate could impact pregnancy outcome in this metabolic high-risk population. Thus, for the current analysis we focus on this unconsidered aspect and aim to evaluate differences in serum lipids in pregnant women after bariatric surgery compared to obese mothers and controls with normal weight as well as to further investigate their impact on birth weight.

\section{Materials and Methods}

\section{Study Participants and Experimental Methods}

This prospective cohort study was performed at the Division of Obstetrics and Fetomaternal Medicine and Division of Endocrinology and Metabolism, Medical University of Vienna, from April 2014 to February 2016. Details of the study protocol and study participants with detailed characterization of glucose metabolism were reported in Göbl et al. [3]. In short, as summarized in a flow chart of our study population (Fig. 1), we included 25 pregnant women with a history of RYGB in addition to 19 obese (preconception BMI $\geq 35$ ) and 19 normal-weight controls (preconception $\mathrm{BMI}<25$ ) between the 24th and 28th weeks of gestation. Women with infectious diseases, such as hepatitis B or C, human immunodeficiency virus (HIV) as well as any liver or renal disease were excluded from participation. Further, women with a history of other surgical procedures than laparoscopic RYGB surgery were not eligible for inclusion in the bypass subgroup. Moreover, women with insulin treatment before study enrollment were excluded. Participants received a detailed metabolic characterization including routine laboratory assessments, anthropometric measurements and 3-h 75-g oral glucose tolerance test. All examinations were repeated in a subgroup after delivery (3-6 months). Moreover, neonatal biometry was assessed after delivery according to sex and age-adjusted percentiles of the Austrian population (available in 48 singleton pregnancies). 


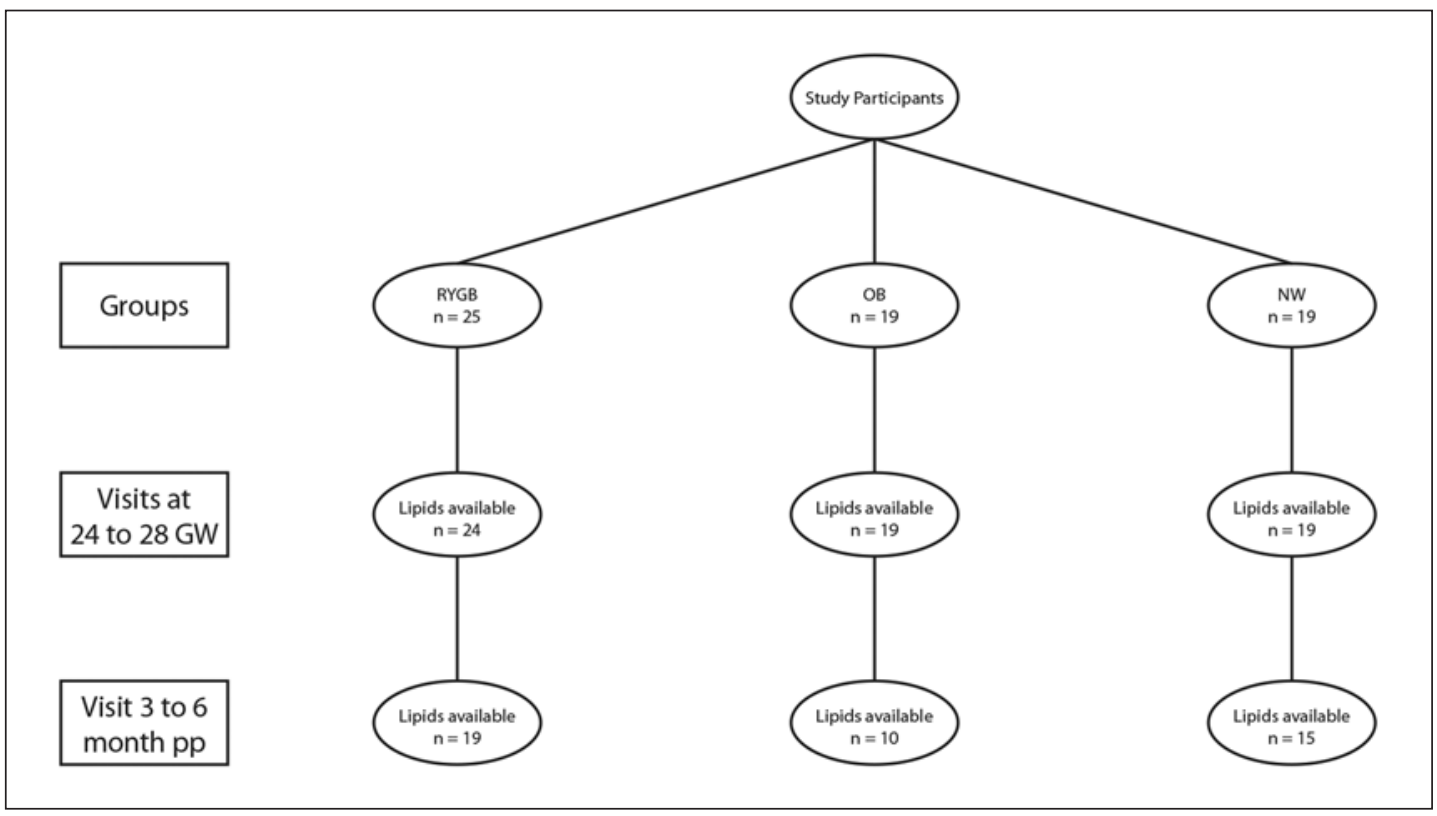

Fig. 1. Flow chart of the study population. GW, gestational week; pp, post partum; OB, obesity; NW, normal weight.

\section{Laboratory Methods}

Serum TG, total cholesterol (TC), high-density lipoprotein C (HDL-C), low-density lipoprotein C (LDL-C) and ultrasensitive C-reactive protein (usCRP) concentrations were evaluated at recruitment once during the 24th-28th gestational week as well as during the second visit within 3-6 months after pregnancy. All parameters were measured according to the international standard laboratory methods at our certified Department of Medical and Chemical Laboratory Diagnostics (http://www.kimcl.at/).

\section{Statistical Analysis}

Continuous variables were summarized by mean and standard deviations or median and interquartile ranges and compared by analysis of variance (ANOVA) and Fisher's protected least significant for difference tests (3 groups) or Student's $t$ test (2 groups). Rank-based inference was used in case of skewed distributed parameters. Associations between continuous variables were examined by Spearman's rank correlation. In order to examine the effect of bariatric surgery independent of actual BMI, we further compared 14 RYGB and 14 BMI-matched women from the control groups by 1:1 nearest-neighbor matching as reported previously [3]. Statistical analysis was performed with R (v3.3.2) and contributing packages [6]. A 2-sided $p$ value $\leq 0.05$ was considered statistically significant.

\section{Results}

Descriptive characteristics of the populations as well as comparisons of serum lipids between subgroups are provided in Table 1. In particular, women with a history of gastric bypass surgery showed lower TC, LDL-C, non-HDL-C and TG concentrations in relation to mothers with preconception obesity as graphically presented in Figure 2. Although affected 
Table 1. Comparison of main characteristics during pregnancy at 24th-28th gestational weeks (median 26, IQR 25-27) and 3-6 months pp between women with a history of Roux-en-Y-gastric bypass (RYGB) and the control groups, i.e. obese expectant mothers with preconception $\mathrm{BMI} \geq 35(\mathrm{OB})$ and normal-weight women with preconception $\mathrm{BMI}<25$ (NW)

\begin{tabular}{|c|c|c|c|c|c|c|c|}
\hline & $n$ & RYGB & $n$ & OB & $n$ & NW & $p$ value \\
\hline Age, years & 25 & $31.9 \pm 7.0$ & 19 & $31.9 \pm 4.8$ & 19 & $30.8 \pm 5.7$ & 0.802 \\
\hline $\mathrm{BMI}$ & 23 & $28.6 \pm 5.0$ & 19 & $38.0 \pm 2.9^{\mathrm{a}}$ & 19 & $21.7 \pm 2.1^{\mathrm{b}}$ & $<0.001$ \\
\hline usCRP, mg/dL & 24 & $0.29 \pm 0.22$ & 19 & $0.82 \pm 0.51^{\mathrm{a}}$ & 18 & $0.32 \pm 0.24$ & $<0.001$ \\
\hline Total cholesterol, mg/dL & 24 & $199.7 \pm 36.9$ & 19 & $227.7 \pm 47.0^{\mathrm{a}}$ & 19 & $256.1 \pm 45.4^{\mathrm{b}}$ & $<0.001$ \\
\hline LDL-cholesterol, mg/dL & 24 & $97.2 \pm 24.9$ & 18 & $124.4 \pm 42.2^{\mathrm{a}}$ & 19 & $141.7 \pm 39.4^{\mathrm{b}}$ & $<0.001$ \\
\hline NHDL-cholesterol, mg/dL & 24 & $127.1 \pm 31.8$ & 18 & $163.2 \pm 46.1^{\mathrm{a}}$ & 19 & $179.0 \pm 48.2^{\mathrm{b}}$ & $<0.001$ \\
\hline HDL-cholesterol, mg/dL & 24 & $72.6 \pm 14.8$ & 18 & $63.8 \pm 12.7$ & 19 & $77.1 \pm 24.5$ & 0.081 \\
\hline Triglycerides, mg/dL & 24 & 127 (117-161) & 19 & $207(140-227)^{a}$ & 19 & 159 (129-183) & 0.020 \\
\hline $\mathrm{pp}$ total cholesterol, $\mathrm{mg} / \mathrm{dL}$ & 19 & $159.9 \pm 31.0$ & 10 & $182.8 \pm 24.7$ & 15 & $199.9 \pm 36.2^{\mathrm{b}}$ & 0.003 \\
\hline pp LDL-cholesterol, mg/dL & 19 & $81.2 \pm 32.2$ & 10 & $107.7 \pm 16.0^{\mathrm{a}}$ & 15 & $120.0 \pm 32.0^{\mathrm{b}}$ & 0.001 \\
\hline pp NHDL-cholesterol, mg/dL & 19 & $98.1 \pm 35.7$ & 10 & $132.7 \pm 19.6^{\mathrm{a}}$ & 15 & $139.0 \pm 36.0^{\mathrm{b}}$ & 0.002 \\
\hline pp HDL-cholesterol, mg/dL & 19 & $61.8 \pm 9.2$ & 10 & $50.1 \pm 12.0^{\mathrm{a}}$ & 15 & $60.9 \pm 13.8$ & 0.030 \\
\hline $\mathrm{pp}$ triglycerides, $\mathrm{mg} / \mathrm{dL}$ & 19 & $76(62-104)$ & 10 & $112(84-164)$ & 15 & $99(61-129)$ & 0.153 \\
\hline
\end{tabular}

Data are means and standard deviations or medians and interquartile ranges (IQR) for patients with RYGB, obese women (OB) and normal-weight controls (NW) during pregnancy and postpartum (pp). BMI, body mass index; usCRP, ultrasensitive C-reactive protein; LDL, low-density lipoprotein; HDL, high-density lipoprotein; NHDL, non-high-density lipoprotein. $p$ values: test for global hypothesis (ANOVA; rank-based inference for triglycerides): RYGB vs. obese vs. normal weight, $p<0.05$. ${ }^{\mathrm{a}} p<0.05$ : RYGB vs. obese; ${ }^{\mathrm{b}} p<0.05$ : RYGB vs. normal weight.

by higher BMI, women with former RYGB even showed lower levels of TC and LDL-C and nonHDL-C compared to normal-weight controls. Moreover, usCRP as an indicator of subtle inflammation was markedly decreased in women with RYGB as they achieved concentrations almost comparable to normal-weight controls.

In the matched case-control analysis, the final group showed comparable BMI (RYGB: median 28.7 [IQR 28.0-36.5] vs. control median 28.8 [IQR 27.4-37.7], $p=0.974$ ). This comparison showed distinct differences in TC, LDL-C, non-HDL-C and usCRP concentrations between both groups (RYGB vs. control; LDL-C: $96.0 \pm 26.2$ vs. $139.0 \pm 54.61 \mathrm{mg} / \mathrm{dL}, p=0.013$; TC: $202.4 \pm 36.7$ vs. $248.6 \pm 64.4 \mathrm{mg} / \mathrm{dL}, p=0.028$; non-HDL-C: $127.8 \pm 33.8$ vs. $173.1 \pm 57.3$ $\mathrm{mg} / \mathrm{dL}, p=0.017$; usCRP: $0.25 \pm 0.18$ vs. $0.47 \pm 0.31 \mathrm{mg} / \mathrm{dL}, p=0.032$ ).

Maternal TC $(\rho=0.59, p=0.021)$, LDL-C $(\rho=0.71, p=0.003)$, non-HDL-C $(\rho=0.59, p=$ 0.021 ) levels but not HDL-C or TG were associated with percentiles of birth weight (available in 16 singleton pregnancies affected by RYGB). None of the neonates born to women with a history of RYGB were large for gestational age but we observed in total 3 cases $(18.8 \%)$ of small-for-gestational-age offspring in this group. Detailed information on differences in neonatal outcome parameters between the groups are provided as supplementary material (online suppl. Table S1; see www.karger.com/doi/10.1159/000504176 for all online suppl. material). After delivery, lipid levels decreased significantly in all women; however, TC concentrations showed a more attenuated decrease in mothers of the RYGB group as compared to controls (median decrease in RYGB $22 \mathrm{mg} / \mathrm{dL}$ [IQR 18-55] vs. normal-weight subjects 61 $\mathrm{mg} / \mathrm{dL}$ [IQR 48-76], $p=0.003$ ). Comparable findings were observed for LDL-C. 


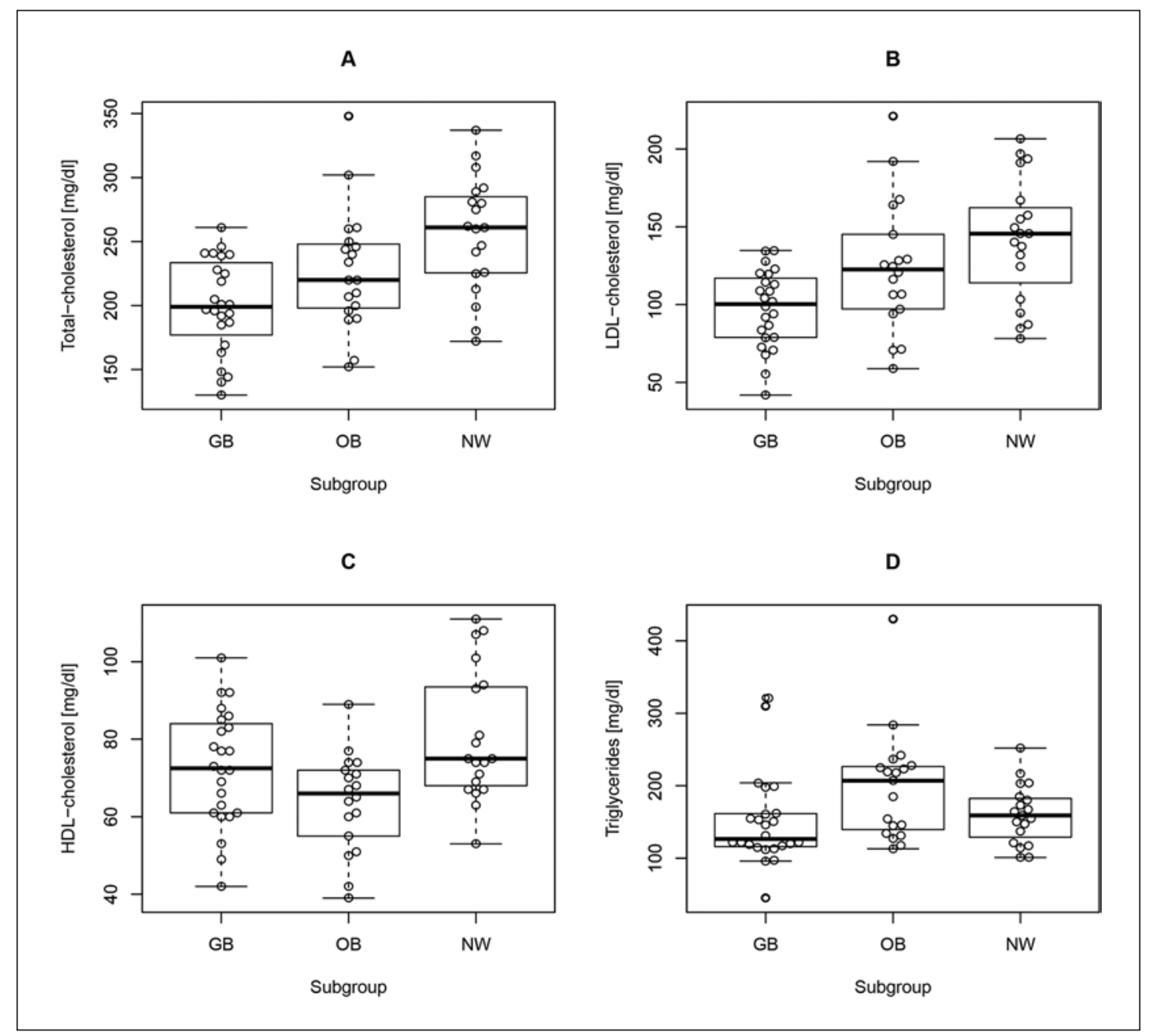

Fig. 2. Box plot of total cholesterol (A), low-density lipoprotein (LDL) C (B), high-density lipoprotein (HDL) $\mathrm{C}(\mathbf{C})$ and triglycerides (D) at baseline in different subgroups, i.e. women after gastric bypass (GB), obese controls (OB) and normal-weight pregnancies (NW).

\section{Discussion/Conclusion}

Pregnant women after bariatric surgery have significant differences in lipids during gestation that distinguish them from obese women as well as normal-weight controls, and this is, as far as we know, the first study providing evidence from prospective data among this special population. The impact of weight change becomes obvious as TC, LDL-C, non-HDL-C and TG are decreased in pregnancies after bariatric procedures compared to those with preconception obesity. Observations in nonpregnant populations suggest favorable effects of bariatric surgery on obesity-related comorbidities, particularly by improvement of the metabolic profile [7]. However, metabolism during pregnancy is disposed to adaptations that are essential for the development and growth of the fetus, and these comprise specific patterns of lipids during gestation [8]. We formerly showed that lipid constellation during pregnancy is altered in obese women compared to normal-weight mothers [4]: hereby maternal obesity was affected by hypertriglyceridemia and low HDL-C. These alterations persisted with progressing pregnancy and were further accompanied by distinguishing LDL-C and TC trajec- 
tories [4]. Besides hyperglycemia, maternal lipids are assumed as main substrates that in excess could contribute to the development of macrosomia, one of the most relevant perinatal complications of obesity as well as to other several adverse pregnancy outcomes, i.e. preeclampsia or preterm delivery [8]. In well-controlled gestational diabetes mellitus (GDM) pregnancies, maternal TG and nonesterified fatty acids were positively correlated with neonatal weight and fat mass [9]. We previously demonstrated that deviations in lipid trajectories are associated with a degree of maternal obesity independent of GDM status [4].

Butalthough bariatric interventions ameliorate the general risk situation for the expectant mother, contrary effects become more apparent that are rather characterized by nutritional deficiencies and consequently higher risk for small-for-gestational-age offspring [1]. In a recent systematic review, it was summarized that malabsorptive procedures are associated with increased odds of small-for-gestational-age neonates but a decrease in odds of being large for gestational age, whereas restrictive procedures were not [10]. In our study 3 neonates $(18.9 \%)$ born to women after RYGB were small for gestational age. There is one retrospective analysis referring to intrauterine fetal growth after maternal gastric bypass surgery, in which the authors observed a continuous and distinctive decline during the course of pregnancy beginning with the second trimester with significant lower birth weight and placental weight at birth compared to BMI-matched controls [11]. The etiology behind this observation of abnormal fetal growth remains to be elucidated.

In our pregnant population, women with former RYGB showed more decreased levels of TC and LDL-C and non-HDL-C compared to normal-weight controls even though they remained at higher BMI after bariatric intervention, and these parameters were further associated with the offspring's birth weight. Differences remained similar when compared to BMI-matched controls. Further analyses showed that all observed effects were independent of the actual BMI or obesity status. Reduction in body fat mass may have the most relevant impact on serum lipids but also alternative mechanisms are assumed behind these changes such as alterations in lipid metabolism or gastrointestinal lipid absorption affecting bile acid metabolism, as well as changes in intestinal microbiome or in gut hormones [1, 2]. Moreover, compared to other procedures RYGB seems to have a peculiar effect by additionally reducing the absorption of cholesterol $[1,12]$. Another aspect that has to be considered is the change in insulin response after intake of food possibly due to bariatric rearrangements of intestinal anatomy $[3,13]$. In this relation, we previously demonstrated that women after bariatric surgery showed exaggerated insulin release early after ingestion of glucose during the diagnostic oral glucose tolerance test [3]. This increase in insulin action may additionally impact the lipid pattern by reduction of lipolysis. Of note, in obese women with GDM, the rate of macrosomia was lowered in cases receiving insulin therapy compared to the normal-weight reference group [14].

Another indicator for metabolic improvement is the decrease in usCRP in the postbariatric group compared to obese mothers in terms of chronic subclinical inflammation. Although it appears that women after bariatric intervention achieve an even better metabolic risk profile compared to normal-weight pregnancies, they actually show a deviation of substrates that might be essential for physiologic supply during gestation.

Our study provides the strengths of a prospective design examining a special study population, which has up to now been only limitedly characterized by retrospective register studies although emphasized as a growing challenge in obstetrics. This aspect has also to be considered regarding our limitation of a relatively small sample size and the number of losses to followup after pregnancy, which is additionally subjectable to the general low adherence level to postpartum screening that is a common limitation in similar studies [15]. Data are restricted only to one measurement during pregnancy, but with explorative intent we firstly investigated differences in lipids in this specific obstetric cohort in comparison to two control groups 
Bozkurt et al.: Lipids in Pregnancy after RYGB

with normal-weight and obese women. However, these are preliminary results which indicate that differences in trajectories of lipids during the course of gestation should be addressed in future studies.

The rapid decrease in lipids after delivery in normal-weight women might picture the subsequent attenuation to the higher demand during pregnancy - a process that seems to be deteriorated in women with former bariatric surgery. Pregnancy-related changes could promote nutritional deficiencies after bariatric surgery and bear a risk for restriction in fetal development, which should not be neglected despite the much healthier maternal health status and the associated benefits for the offspring. Thus, it becomes of importance to understand the precise consequences of these imbalances for mother and child in order to maximize the benefit of bariatric surgery and to develop adequate guidelines for the prevention of longterm sequelae for the mother and child, which warrant further evidence.

\section{Statement of Ethics}

This study was approved by the Ethics Committee of the Medical University of Vienna and performed in accordance with the Declaration of Helsinki. All participants gave written informed consent to participate in this study.

\section{Disclosure Statement}

The authors have no conflicts of interest to declare.

\section{Author Contributions}

C.S.G. and A.K.-W. designed research. Patient recruitment was performed by M.L. and W.E. Statistical analysis, calculations and data interpretation were performed by C.S.G. and L.B. The manuscript was written by L.B. C.S.G., W.E. and A.K.-W. reviewed and edited the manuscript. All authors have seen and approved the submission of this version of the paper and take full responsibility for the paper.

\section{References}

1 Busetto L, Dicker D, Azran C, Batterham RL, Farpour-Lambert N, Fried M, et al. Obesity Management Task Force of the European Association for the Study of Obesity Released "Practical Recommendations for the PostBariatric Surgery Medical Management”. Obes Surg. 2018 Jul;28(7):2117-21.

2 Johansson K, Cnattingius S, Näslund I, Roos N, Trolle Lagerros Y, Granath F, et al. Outcomes of pregnancy after bariatric surgery. N Engl J Med. 2015 Feb;372(9):814-24.

3 Göbl CS, Bozkurt L, Tura A, Leutner M, Andrei L, Fahr L, et al. Assessment of glucose regulation in pregnancy after gastric bypass surgery. Diabetologia. 2017 Dec;60(12):2504-13.

4 Bozkurt L, Göbl CS, Hörmayer AT, Luger A, Pacini G, Kautzky-Willer A. The impact of preconceptional obesity on trajectories of maternal lipids during gestation. Sci Rep. 2016 Jul;6(1):29971.

5 Leutner M, Klimek P, Göbl C, Bozkurt L, Harreiter J, Husslein P, et al. Glucagon-like peptide 1 (GLP-1) drives postprandial hyperinsulinemic hypoglycemia in pregnant women with a history of Roux-en-Y gastric bypass operation. Metabolism. 2019 Feb;91:10-7.

6 R Development Core Team. R: a language and environment for statistical computing. R Foundation for Statistical Computing (2015), Vienna, Austria [cited 2017 Dec 25]. Available from: http://www.R-project.org/

7 Sjöström CD, Lissner L, Wedel H, Sjöström L. Reduction in incidence of diabetes, hypertension and lipid disturbances after intentional weight loss induced by bariatric surgery: the SOS Intervention Study. Obes Res. 1999 Sep; $7(5): 477-84$. 
8 Herrera E, Ortega-Senovilla H. Lipid metabolism during pregnancy and its implications for fetal growth. Curr Pharm Biotechnol. 2014;15(1):24-31.

9 Herrera E, Ortega-Senovilla H. Implications of Lipids in Neonatal Body Weight and Fat Mass in Gestational Diabetic Mothers and Non-Diabetic Controls. Curr Diab Rep. 2018 Feb;18(2):7.

10 Akhter Z, Rankin J, Ceulemans D, Ngongalah L, Ackroyd R, Devlieger R, et al. Pregnancy after bariatric surgery and adverse perinatal outcomes: A systematic review and meta-analysis. PLoS Med.2019 Aug;16(8):e1002866.

11 Feichtinger M, Falcone V, Schoenleitner T, Stopp T, Husslein PW, Eppel W, et al. Intrauterine fetal growth delay during late pregnancy after maternal gastric bypass surgery. Ultraschall Med. 2018 Oct. German. Available from: https://doi.org/10.1055/a-0651-0424

12 Carswell KA, Belgaumkar AP, Amiel SA, Patel AG. A Systematic Review and Meta-analysis of the Effect of Gastric Bypass Surgery on Plasma Lipid Levels. Obes Surg. 2016 Apr;26(4):843-55.

13 Mosinski JD, Kirwan JP. Longer-Term Physiological and Metabolic Effects of Gastric Bypass Surgery. Curr Diab Rep. 2016 Jun;16(6):50.

14 Langer O, Yogev Y, Xenakis EM, Brustman L. Overweight and obese in gestational diabetes: the impact on pregnancy outcome. Am J Obstet Gynecol. 2005 Jun;192(6):1768-76.

15 Hamel MS, Werner EF. Interventions to improve rate of diabetes testing postpartum in women with gestational diabetes mellitus. Curr Diab Rep. 2017 Feb;17(2):7. 